\title{
9 Considerations when using longitudinal statistical models to study ageing
}

\author{
Annie Robitaille and Graciela Muniz Terrera
}

\section{Research methods}

Longitudinal statistical models are presented to examine the ageing process. Approaches discussed include the latent growth curve model, multivariate latent growth curve model, latent growth curve model with a time-varying covariate, latent class analysis, growth mixture model, multi-state model and change point model.

\section{Methodological lessons learned}

This chapter highlights the increased access to longitudinal data sources and the variety of different longitudinal models of change available to researchers interested in ageing. These data sources and longitudinal models provide researchers with an opportunity to answer complex research questions related to ageing and within-person change. Researchers should devote efforts to choosing the data source and longitudinal model that matches most closely to their research questions because different models lead to different results.

\section{Summary}

In order to understand the ageing process, it is important that the same older adults are assessed at two or more occasions over time. Even though there has been an increase in longitudinal data and advances in statistical modelling, most research is still based on between-person differences rather than on within-person change. This chapter will discuss statistical models made possible with repeated measurements (e.g., latent growth curve model, growth mixture model) that are important for assessing changes that occur within older adults. Important considerations when using longitudinal statistical models will be discussed (e.g., using longitudinal data that matches your research question, gaining access to data, features of study design such as time elapsed between measurement occasions and number of occasions, comparison across countries). We hope this chapter will make it easier for researchers to use longitudinal approaches to their utmost potential.

\section{Introduction}

It is well known that the ageing process is complex, requiring an understanding of multiple biological, social, physical, neurological and psychological factors over an individual's lifetime. In order to understand the ageing process, it is important to examine how these numerous biopsychosocial factors change and interact over an extended period as the effect of these factors on the ageing process can take years to manifest. Looking at the effect of 
these factors at only one point in time only provides a snapshot of the ageing phenomenon. It fails to provide much needed information about the trajectories and transitions that older adults go through and its determinants.

The importance of understanding the complexity of various ageing phenomena has resulted in increased funding worldwide for large-scale longitudinal ageing studies that follow the same individuals over time (Woodard, 2017). These studies vary in the age of the targeted older adults (e.g., some studies follow individuals from birth to older adulthood, others start following individuals in older adulthood, and others specifically focus on the oldest age group), the number of repeated occasions available (e.g., some only include three time points and others include many more), the time intervals between studies (e.g., some collect information every year and others every three years) and the variables included (e.g., blood samples, psychological measures, neurological measures, cognitive measures); providing researchers with increased opportunities to select longitudinal studies that most closely answer their research questions. For example, a researcher interested in understanding the cognitive ageing process from normal cognitive ageing to dementia would want to select a study that follows older adults across numerous repeated waves of data collection over many years with valid measures of cognitive ageing and dementia.

This increase in longitudinal studies has also resulted in more procedures put in place that facilitate access to data (e.g., GAIIN, Gateway to ageing, DPUK). For example, numerous data sources can now be accessed at data centers or online with data request forms. The increased access to longitudinal studies has also led to an increase in research networks aimed at facilitating the integration of numerous longitudinal studies (e.g., Integrative Analysis of Longitudinal Studies of Ageing and Dementia). These networks make it easier for researchers to run statistical analysis across multiple longitudinal studies on ageing thus facilitating study replication and the examination of cross-cultural and cohort effects (Hofer and Piccinin, 2009, 2010). In addition, linkages between different data sources (e.g., national longitudinal studies, electronic medical records, long-term care data) is now also possible allowing researchers to complement their longitudinal studies with information that was not directly collected but that is important to understanding the ageing process.

This surge is longitudinal studies and the more recent ease of access to this data has also resulted in an increased use of longitudinal modeling approaches. Traditionally, psychological research has focused mostly on cross-sectional models that examine individual differences (between-person differences; e.g., how older adults of different ages differ from each other on cognitive functioning) rather than on longitudinal models that allow the examination of how individuals change over time (within-person change). More recently, researchers are requesting more longitudinal designs to better understand the within-person processes of ageing given that within-person research questions answered using crosssectional designs can lead to biased results (Molenaar and Campbell, 2009).

Developments in advanced statistical models and computer software (e.g., Mplus, R, $\mathrm{Mx}$ ) for the modeling of one or more variables collected over various time points allow researchers to answer complex research questions about ageing-related trajectories and the dynamic relationship between selected developmental processes. These models also allow researchers to examine the extent to which results based on cross-sectional designs align with those of longitudinal designs. These statistical advancements have resulted in more than one analytical technique to study longitudinal change (see Bollen and Curran, 2006; Duncan, Duncan and Strycker, 2006; Grimm, 2007; Hoffman, 2015; Muniz-Terrera et al., 2016; Singer and Willett, 2003) each of which can provide different insight into the 
trajectories of older adults. Using statistical techniques for longitudinal data (e.g., latent growth curve modeling and multilevel modeling) allows the identification of average trajectories of change (initial level and rate of change) and variability around the initial level and rate of change (interindividual heterogeneity) both of which are key in understanding the ageing process. The availability and complexity of these analytical techniques and the fact that new developments in the analysis of change are constantly evolving means that researchers must choose the most appropriate model even though they may not be aware of the different longitudinal models available to study ageing.

The purpose of this chapter is to provide researchers with an overview of longitudinal studies available to study ageing and review some of the longitudinal data approaches to studying the trajectories of ageing. This chapter is not meant as an exhaustive list of all longitudinal studies and data analysis methodologies but rather as an overview of some of these to highlight the range of studies available around the world and the various different longitudinal data approaches to analyze the data from these studies. More specifically, univariate and multivariate latent growth curve models, the latent growth curve models with a time-varying covariate, growth mixture models, change-point models and multi-state models will be discussed. These statistical models were chosen given their significance in understanding the trajectories and transitions of the ageing process. While there is overlap between many of these models, each still displays important distinctions which affect the research questions each can answer. We hope to provide readers with an understanding of the different statistical models and the research questions that can be answered by each of them.

\section{Longitudinal studies}

Before discussing the statistical models available to studying ageing trajectories, we felt it was important to highlight briefly some of the longitudinal studies available around the world. As mentioned in the introduction of this chapter, there has been an increase in accessibility of longitudinal studies on ageing worldwide (Woodard, 2017) and it is imperative that more researchers become aware of these data and their importance in further understanding the ageing process. These studies are imperative as cross-sectional studies only provide a snapshot of the lives of older adults. Some of the large-scale longitudinal studies on ageing include the Seattle Longitudinal Study (SLS), Baltimore Longitudinal Study of Ageing (BLSA), Victoria Longitudinal Study (VLS, Hultsch et al., 1998), English Longitudinal Study of Ageing (ELSA, Clemens et al., 2019), Origins of Variance in the OldestOld: Octogenarian Twins (OCTO-Twin, Johansson et al., 2004; McClearn et al., 1997), Survey of Health, Ageing and Retirement in Europe (SHARE, Borsch-Supan et al., 2013), Longitudinal Ageing Study Amsterdam (LASA; Hoogendijk et al., 2016; Huisman et al., 2011), Rush Memory and Ageing Project (MAP; Bennett et al., 2012; Bennett et al., 2005), Wisconsin Longitudinal Study (WLS, Hauser and Weir, 2010), Swedish Adoption/Twin Study of Ageing (Finkel and Pedersen, 2004; Pedersen, Lichtenstein and Svedberg, 2002), Canberra Longitudinal Study (Christensen et al., 2004) and Health and Retirement Study (HRS, Sonnega et al., 2014). This is a list of some of the longitudinal studies on ageing that are available, as an exhaustive list of all longitudinal studies was not the objective of this chapter.

Researchers interested in longitudinal studies are encouraged to examine these studies more closely to assess whether they would be appropriate for their research questions, as these studies all vary in the variables they measure, the number of repeated assessments 
they completed, the age range of participants, sample size and the time intervals between assessments. These are important to consider as they can have an impact on the interpretation of results. For example, a researcher interested in examining trajectories of people with dementia would not want to select a longitudinal study with repeated assessments at fiveyear intervals. Shorter data collection intervals would be needed to capture crucial information about dementia trajectories. Similarly, a researcher interested in trajectories of cognitive functioning would preferably want data for four or more repeated occasions in order to examine nonlinear trajectories.

In addition to these studies, researchers in the field of ageing should also consider other sources of longitudinal data such as administrative data. For example, in Canada, data from electronic medical records is available through the Canadian Primary Care Sentinel Surveillance Network (CPCSSN; http://cpcssn.ca/about-cpcssn/). There is also the InterRAI (www.interrai.org) data which has been adopted worldwide. For example, in Canada the InterRAI data is used in long-term care homes to assess demographic information and clinical and functional characteristics of residents (e.g., cognitive and physical function, psychosocial well-being, health conditions). Repeated assessments are done every three months for the duration of the resident's stay in long-term care, making this data relevant to researchers interested in understanding the trajectories of people living in long-term care homes. For example, we used this data to examine the longitudinal relationship between dementia-related challenging behaviors (e.g., vocal disruption, physical aggression) and cognitive functioning in long-term care homes (Robitaille, Garcia and McIntosh, 2015).

\section{Longitudinal data networks}

Replication of longitudinal research is necessary to protect against uncritical acceptance of empirical results. Research findings and conclusions often vary across independent studies addressing the same topic. A number of reasons are possible for the discrepancies such as differences in measures, analysis and covariates used and cultural differences between populations. The wide variety of possible reasons for the discrepancies makes it difficult to untangle the real reason for the between-study variability. Longitudinal data networks make it easier for researchers to replicate longitudinal research across multiple studies. Using a coordinated analytical approach, researchers can take steps to insure a common protocol across all studies That is, the same statistical models (e.g., a multivariate latent growth curve model is used across all studies) can be used with the same variables (e.g., a Mini Mental State Examination (MMSE) and a measure of grip strength are used to examine the longitudinal relationship between cognitive and physical functioning across all studies) and covariates (e.g., age, gender, years of education are used across all studies) across multiple longitudinal studies. For examples of studies that have used a coordinated approach see, Brown et al. (2012), Duggan et al. (2019), Lindwall et al. (2012), Mitchell et al. (2012), Piccinin et al. (2013), Robitaille et al. (2018a) and Zammit et al. (2019).

Longitudinal data analysis networks include the Integrative Analysis of Longitudinal Studies of Ageing and Dementia (IALSA; www.maelstrom-research.org/mica/network/ ialsa), Promoting Mental Well-being and Healthy Ageing in Cities (MINDMAP, Beenackers et al., 2018) and Ageing Trajectories of Health: Longitudinal Opportunities and Synergies (ATHLOS; http://athlosproject.eu/). Researchers interested in data harmonization or coordinated data analysis should also take a look at Maelstrom Research (www. maelstrom-research.org/). It provides access to an international team of experts in longitudinal data analysis, open-source software, harmonization platforms and free access to the 
Maelstrom Catalogue (i.e., metadata catalog) which provides researchers with easy access to information about existing longitudinal research networks and a large number of longitudinal studies (e.g., study design, variables collected and whether bio specimens were collected, age of participants, number of data collection waves, country where data is collected).

\section{Longitudinal statistical approaches}

Throughout this section, we refer mostly to latent curve models, which are based on the structural equation modeling framework. However, for those who prefer multilevel analyses, multilevel models for longitudinal data in which observations are nested within individuals can also be used as both are equivalent (Hox, 2010).

\section{Latent growth curve model}

Latent growth curve models allow researchers to examine trajectories (i.e., intercept and slope) of repeated measures and examine the effect of various variables on these trajectories (Bollen and Curran, 2006; Curran, Obeidat and Losardo, 2010). For example, Piccinin et al. (2013) examined the association between age, sex and education with MMSE scores using a time in study latent growth models across six studies of ageing. That is, latent growth curve models allow researchers to examine mean trajectories (e.g., what is the average initial level of cognitive functioning and how does cognition change as a function of time) and the role of various variables (e.g., chronic conditions, education) on the intercept (e.g., levels of cognitive functioning at baseline) and the slope (e.g., rate of cognitive decline) to examine whether some older adults demonstrate more rapid decline compared to others. Latent growth curve models can either be linear, in which the rate of change is assumed to be constant across all repeated occasions, or nonlinear, in which a more complex rate of change (e.g., quadratic trajectory) is modeled. Researchers interested in modeling an ageing process that is expected to show accelerated or decelerated decline across time (e.g., memory) should model a nonlinear trajectory over time, although this will depend on the number of measuring occasions. In addition to using age or time in study as the time metric, latent growth curve models can also be used to model time to death and time to disease onset (e.g., Cadar et al., 2016).

\section{Multivariate latent growth curve model (MLGC)}

The MLGC model is an extension of the univariate growth curve model (Bollen and Curran, 2006; Curran, Obeidat and Losardo, 2010). It estimates the random trajectory of two variables (known to be correlated) simultaneously and examines how these correlate together over time. What makes this model interesting is that it allows for the examination of the correlation between the outcome's intercepts (intercept-intercept correlation), slopes (slope-slope correlation) and occasion-specific residuals (OSRs; OSR-OSR correlation). The slope-slope correlation examines whether people who decline more quickly than average on one variable (e.g., processing speed) also demonstrate a higher than average rate of decline for another variable (e.g., visual spatial ability). On the other hand, the OSR-OSR relationship is within-person, time-specific deviations/fluctuations around people's long-term developmental trends on some outcome (Hofer et al., 2009; Martin and Hofer, 2004). Therefore, this approach examines whether change for a specific individual 
at a specific occasion is associated with another variable at a matched occasion (Curran and Bauer, 2011; Hofer et al., 2009; Hoffman and Stawski, 2009). For example, it examines whether within-person variations from each individual's mean trajectory of processing speed is associated with occasion-specific residuals of the visual spatial ability measure.

While both approaches (slope-slope and OSR-OSR correlation) appear similar, they answer two rather different questions and relying on one approach over another can result in different conclusions about the relationship between both developmental variables (Hofer et al., 2009; Sliwinski and Mogle, 2008). Even though memory and social participation may both go up or down together at the overall trend level, they may not necessarily share the same transient deviations between shorter time intervals. To date, the majority of research has focused on associations between mean trajectories rather than relationships between occasion-specific residuals (Hofer et al., 2009).

In addition to providing estimates of the relationship between the intercepts, the slopes and the OSRs, this model also allows for the examination of the initial level and rate of change of each variable independently. Given that only correlations are estimated between both variables, estimating two independent univariate growth curve models would give identical results. See Muniz-Terrera et al. (2016), Praetorius Björk, Johansson and Hassing (2016), Robitaille, Garcia and McIntosh (2015), Robitaille et al. (2012) and Sliwinski, Hofer and Hall (2003) for papers where bivariate growth curve models were applied.

Another possibility is to estimate the structure of the MLGC model by regressing the slope of $y$ on the slope of $x$ and the intercept of $y$ on the intercept of $x$ (Bollen and Curran, 2006). For example, this model implies that changes in visual spatial ability may be explained by processing speed and therefore aligns more closely with the theoretical basis of the role of processing speed on cognitive ageing. Unlike the MLGC model which includes two outcome variables, this model includes a predictor and an outcome variable. The initial level and rate of change for visual spatial ability now accounts for what is explained by processing speed and is therefore no longer equivalent to results that would be obtained from a univariate latent growth curve model. Rather, the initial level and rate of change of visual spatial ability is net of the effect of processing speed. The initial level and rate of change of processing speed remains identical to the aforementioned model. For now, correlations are still included between the occasion-specific scores for the predictor and the outcome but as we will see in the next section, regressions can also be estimated.

\section{Latent growth curve model with a time-varying covariate (TVC)}

The latent growth curve model with a TVC is similar to the directional MLGC model in that only one outcome and one predictor variable is modeled and is therefore only pertinent to use in situations where there is some theoretical basis for having one variable predict the other. Unlike the directional MLGC model, the TVC model does not necessarily include a random slope for the TVC. That is, the predictor variable can be modeled as a variable that fluctuates over time rather than changing systematically over time.

In the TVC model, repeated measures of cognitive functioning are regressed on processing speed at each occasion to take into account the time-specific fluctuations of processing speed in explaining cognitive functioning (e.g., memory, visual spatial ability) above and beyond the changes predicted by the general growth trajectory. In addition, the intercept of the outcome variable (e.g., cognitive test) is regressed on the intercept of the TVC (e.g., processing speed) which provides between-person information and, most importantly, disaggregates within- and between-person effects. The intercept and rate of change of the 
outcome is also net of the effect of the TVC. That is, instead of controlling for the effect of the TVC (e.g., processing speed) at baseline only, the effect at each occasion is being controlled. Time-invariant predictors (e.g., initial age and sex) are interpreted somewhat differently in TVC models in that the role of initial age in predicting cognitive functioning is net of the effects of processing speed (Bollen and Curran, 2006).

Disaggregating both effects is especially important in order to avoid confounding both effects which could result in biased model estimates (Curran and Bauer, 2011; Hoffman, 2015; Hoffman and Stawski, 2009; Wang and Maxwell, 2015). Unlike time-invariant covariates which remain stable over occasions, time-varying covariates, such as processing speed, are variables that change over time. In addition to changing over time within the same individual, different individuals also have varying overall levels of the TVC when compared to others. For example, although processing speed is expected to decrease in older adults with ageing, some individuals inherently have higher levels of processing speed compared to others. Therefore, as time-varying covariates, in their raw form, typically contain both between-person and within-person sources of variance, it is essential to disaggregate these effects (Curran and Bauer, 2011; Curran et al., 2014; Hoffman and Stawski, 2009; Wang and Maxwell, 2015). In order to disentangle the within-person effect from the between-person effect, in addition to being included at the within-person level (level 1), processing speed is included at the between-person level (level 2) by including its random intercept in the model. The intercept of the outcome variable is then regressed on the TVC in order to extract information about the between-person effect of the TVC on the outcome. See Bielak etal. (2012), MacDonald et al. (2003) and Sliwinski and Buschke $(1999,2004)$ for examples where TVC models were applied to cognitive ageing research. An extension of this model would be to add a random slope to the TVC if it is believed that the TVC changes as a function of time/age. This would be very similar to the directional MLGC model with the additional prediction between occasion-specific predictor and matching occasion-specific outcome.

\section{Latent class analysis (LCA) and growth mixture model (GMM)}

Developments in the analysis of longitudinal data have resulted in recent studies focused on better understanding heterogeneity in the ageing-related trajectories. Two such developments, LCA and GMM, allow for the identification of different classes of individuals who cluster together thus identifying more than one trajectory within the population (Asparouhov and Muthén, 2014; Berlin, Parra and Williams, 2014; Grimm and Ram, 2009; Jung and Wickrama, 2008; Liu and Hancock, 2014; Muthén, 2004; Muthén and Shedden, 1999). Although the growth curve models mentioned above do evaluate heterogeneity, this approach goes further by examining whether sufficient heterogeneity exists to form different trajectories that group together into distinct classes. Unlike latent growth curve models, these models have an added latent categorical variable (also referred as a trajectory class variable) in order to model the potential unobserved subpopulation membership (Berli, Parra and Williams, 2014; Muthén, 2002, 2004; Muthén and Shedden, 1999). Therefore, LCA and GMM can be used to understand more precisely the trajectories of ageingrelated processes beyond the average trends, thus providing opportunities for researchers not only to examine the role of predictors on the intercept and rate of change, but also examine the role of predictors on class membership.

An important distinction between LCA and GMM is that GMM models are more flexible than latent class models. That is, whereas the GMM model allows for variability 
on each intercept and slope, the LCA intercept and slope are fixed to zero, making the assumption that the trajectories within classes are homogeneous (Berlin, Parra and Williams, 2014; Muthén, 2004; Nagin, 1999). GMM provides estimates of the average trajectory (intercept and slope) and variation for older adults in each latent class (Berlin, Parra and Williams, 2014; Muthén, 2004, 2002; Muthén and Shedden, 1999). GMM provides information about the optimal number of classes and characteristics of each class including the mean intercept and slope, proportion of membership and significant predictors of class membership. The number of classes identified is based on an exploratory approach in that models are fit with increasing number of classes (e.g., one class, two classes, three classes and four classes). The number of classes is based on the fit of the models and the interpretability of the classes (i.e., BIC/AIC values, entropy values, Bayesian Information Criterion, bootstrap likelihood ratio test; Nylund, Asparouhov and Muthén, 2007). A number of studies have used GMM to explore the optimal number of classes for cognitive ageing (Proust and Jacqmin-Gadda, 2005; Small and Bäckman, 2007; Terrera, Brayne and Matthews, 2010; Hayden et al., 2011; Xie, Mayo and Koski, 2011). These studies suggest that different groups of trajectories might better explain change in cognitive functioning and that forcing everyone to fit within the average group might be misrepresentative.

More recently, some studies have modelled multivariate GMM (Robitaille et al., 2018a). Unlike the univariate GMM, the multivariate model includes two outcomes modeled simultaneously allowing for the additional possibility to examine the relationship between the two outcomes within each of the identified classes (e.g., cognition and grip strength). LCA and GMM have important clinical implications in that they can help predict which individuals are at increased risk of being in the low functioning classes.

\section{Multi-state model (MSM)}

Unlike the aforementioned models, multi-state modeling can be used to assess the transitions of older adults across different states such as health related processes of ageing (e.g., dementia, disease) and examine the role of risk factors on the transitions between states (van den Hout, 2017). These models are also referred to as multi-state survival models given that death is included as one of the states. Importantly, these models are flexible in the number of states that can be included and allow for the modeling of transitions even though the exact time of the transition (e.g., exact time of dementia diagnosis) is unknown (van den Hout, 2017). For example, Robitaille et al. (2018b) examined the role of education on transitions through cognitive states and death using a four-state model with State 1 defined as normal MMSE, State 2 as mild MMSE impairment, State 3 as severe MMSE impairment and State 4 defined as death. Robitaille et al. found that a higher level of education was associated with a lower risk of transitioning from normal MMSE to mild MMSE impairment but was not associated with the later transitions. This approach also allows for the estimation of overall and non-impaired life expectancies (van den Hout, 2017). For example, Robitaille et al. (2018b) found that those with higher levels of education and socio-economic status had longer non-impaired life expectancies but that no differences were found on overall life expectancies. Another paper that uses multi-state modeling is Kryscio et al. (2013). Unfortunately, multi-state models are only rarely used in the field of ageing even though the knowledge gained from these models can improve our understanding of risk factor to disease-related processes of ageing, potentially facilitating earlier detection for inclusion in preventative interventions. 


\section{Change point model}

Change point models, a version of random effects (Laird and Ware, 1982) models that permit the identification of the onset of change in rate of decline, have recently become popular in ageing research (Hall et al., 2000; Johnson et al., 2009; Karr et al., 2018; Muniz-Terrera, 2015). They have been used to describe ageing related changes, and accelerated declines that occur in the proximity of death (terminal decline) and prior to the onset of disease. Although change point models can be used to model the onset of accelerated change in any area of research, much of the research literature has focused on accelerated change in cognitive function prior to dementia and death (Karr et al., 2018). In a seminal publication on cognitive ageing research, Hall et al. (2000) identified the onset of faster memory decline in preclinical dementia cases by fitting change point models using profile likelihood and Bayesian estimation. Following this publication, numerous research papers have been published that used change point models (Muniz-Terrera et al., 2013). For example, in an examination of the terminal decline hypothesis, Thorvaldsson et al. (2008) reported onsets of faster decline at 6.6 years before death for verbal ability, 7.8 years before death for spatial ability and 14.8 years for perceptual speed, and Muniz-Terrera et al. (2013) estimated the onset of faster decline at 7.7 years before death for global cognition scores. Most of these papers regarded the change point as a fixed effect, most in preclinical dementia or terminal decline investigations and that described change occurring in two linear phases with an abrupt change. The assumption that the change point is a fixed effect, that is, that all individuals transition from an initial phase of decline to a phase of faster decline at the same distance from death or dementia or age is without doubt, very strong and highly questionable given the observed heterogeneity of the ageing or disease process. More recently, a fewer publications, most of them using Bayesian estimation, have modelled the change point as a random effect (which is essential for identifying individuals for potential treatment) and some have relaxed the assumption of an abrupt change allowing for a transitional period between the two linear phases (Muniz Terrera, van den Hout and Matthews, 2011; Terrera et al., 2014). More researchers need to consider change point models in their study of the ageing process as these models can provide knowledge that can help to plan more timely interventions to delay decline.

\section{Discussion}

The purpose of this chapter was to highlight some considerations that should be taken into account when conducting longitudinal data analysis. As demonstrated, there are numerous data sources available and one cannot overestimate the importance in choosing a longitudinal study that meets your research needs. This chapter also emphasized the variety of different longitudinal models of change available to researchers interested in ageing and highlighted the distinction between these models. The sheer number of different models of change that are available and the variations in parameterization that are possible are making it difficult for researchers to use the best model for their research question. Researchers should devote efforts choosing the model that matches most closely to their research questions because different models lead to different results. Given the wide variety of longitudinal models available, it would be important for researchers to provide an explanation for why one model was chosen and provide a detailed description of the model specifications (e.g., fixed or individually varying time points). Advances in multivariate models of change are allowing researchers to answer complex questions of developmental change. We hope this chapter will make it easier for researchers to use these models to their utmost potential. 


\section{References}

Asparouhov, T. and Muthén, B. (2014). Auxiliary variables in mixture modeling: three step approaches using Mplus. Structural Equation Modeling: A Multidisciplinary Journal, 21(3), 329-341. doi:10.1080/10705511.2014.915181.

Beenackers, M.A., Doiron, D., Fortier, I., Noordzij, J M., Reinhard, E., Courtin, E., Bobak, M., Chaix, B., Costa, G., Dapp, U., Diez Roux, A.B., Huisman, M., Grundy, E.M., Krokstad, S., Martikainen, P., Raina, P., Avendano, M. and van Lenthe, F.J. (2018). MINDMAP: establishing an integrated database infrastructure for research in ageing, mental well-being, and the urban environment. BMC Public Health, 18(1), 158. doi:10.1186/s12889-018-5031-7.

Bennett, D.A., Schneider, J.A., Buchman, A.S., Barnes, L.L., Boyle, P.A. and Wilson, R.S. (2012). Overview and findings from the Rush Memory and Ageing Project. Current Alzheimer Research, 9(6), 646.

Bennett, D.A., Schneider, J.A., Buchman, A.S., Mendes de Leon, C., Bienias, J.L. and Wilson, R.S. (2005). The Rush Memory and Ageing Project: study design and baseline characteristics of the study cohort. Neuroepidemiology, 25(4), 163-175.

Berlin, K.S., Parra, G.R. and Williams, N.A. (2014). An introduction to latent variable mixture modeling (Part 2): longitudinal latent class growth analysis and growth mixture models. Journal of Pediatric Psychology, 39(2), 188-203. doi:10.1093/jpepsy/jst085.

Bielak, A.A.M., Anstey, K.J., Christensen, H. and Windsor, T.D. (2012). Activity engagement is related to level, but not change in cognitive ability across adulthood. Psychology and Ageing, 27(1), 219-228. doi:10.1037/a0024667.

Bollen, K.A. and Curran, P.J. (2006). Latent curve models: a structural equation approach. Hoboken, NJ: Wiley.

Borsch-Supan, A., Brandt, M., Hunkler, C., Kneip, T., Korbmacher, J., Malter, F., Schaan, B., Stuck, S. and Zuber, S. (2013). Data resource profile: the Survey of Health, Ageing and Retirement in Europe (SHARE). International Journal of Epidemiology, 42(4), 992-1001. doi:10.1093/ije/dyt088.

Brown, C.L., Gibbons, L.E., Kennison, R.F., Robitaille, A., Lindwall, M., Mitchell, M.B., Shirk, S.D., Atri, A., Cimino, C.R., Benitez, A., Macdonald, S.W., Zelinski, E.M., Willis, S.L., Schaie, K.W., Johansson, B., Dixon, R.A., Mungas, D.M., Hofer, S.M and Piccinin, A. M. (2012). Social activity and cognitive functioning over time: a coordinated analysis of four longitudinal studies. Journal of Ageing Research. doi:10.1155/2012/287438.

Cadar, D., Stephan, B.C.M., Jagger, C., Johansson, B., Hofer, S.M., Piccinin, A.M. and MunizTerrera, G. (2016). The role of cognitive reserve on terminal decline: a cross-cohort analysis from two European studies: OCTO-Twin, Sweden, and Newcastle 85+, UK. International Journal of Geriatric Psychiatry, 31(6), 601-610. doi:10.1002/gps.4366.

Christensen, H., Mackinnon, A., Jorm, A.F., Korten, A., Jacomb, P., Hofer, S.M. and Henderson, S. (2004). The Canberra longitudinal study: design, aims, methodology, outcomes and recent empirical investigations. Ageing, Neuropsychology, and Cognition, 11(2-3), 169-195. doi:10.1080/ 13825580490511053.

Clemens, S., Phelps, A., Oldfield, Z., Blake, M., Oskala, A., Marmot, M., Rogers, N., Banks, J., Steptoe, A. and Nazroo, J. (2019). English longitudinal study of ageing: waves 0-8, 1998-2017. [data collection]. 30th ed. UK Data Service. SN: 5050.

Curran, P.J. and Bauer, D.J. (2011). The disaggregation of within-person and between-person effects in longitudinal models of change. Annual Review of Psychology, 62, 583-619.

Curran, P.J., Howard, A.L., Bainter, S.A., Lane, S.T. and McGinley, J.S. (2014). The separation of between-person and within-person components of individual change over time: a latent curve model with structured residuals. Journal of Consulting and Clinical Psychology, 82(5), 879-894. doi:10.1037/a0035297.

Curran, P.J., Obeidat, K. and Losardo, D. (2010). Twelve frequently asked questions about growth curve modeling. Journal of Cognition and Development: Official Journal of the Cognitive Development Society, 11(2), 121-136. doi:10.1080/15248371003699969. 
Duggan, E.C., Piccinin, A.M., Clouston, S., Koval, A.V., Robitaille, A., Zammit, A.R., Wu, C., Brown, C.L., Lee, L.O., Finkel, D., Beasley, W.H., Kaye, J., Muniz Terrera, G., Katz, M., Lipton, R.B., Deeg, D., Bennett, D.A., Praetorius Björk, M., Johansson, B., Spiro, A., III, Weuve, J. and Hofer, S.M. (2019). A multi-study coordinated meta-analysis of pulmonary function and cognition in ageing. Journals of Gerontology: Series A, 74(11), 1793-1804. doi:10.1093/gerona/glz057.

Duncan, T.E., Duncan, S.C. and Strycker, L.A. (2006). An introduction to latent variable growth curve modeling: concepts, issues and applications. Mahwah, NJ: Erlbaum.

Finkel, D. and Pedersen, N.L. (2004). Processing speed and longitudinal trajectories of change for cognitive abilities: the Swedish adoption/twin study of ageing. Ageing, Neuropsychology, and Cognition, 11(2-3), 325-345. doi:10.1080/13825580490511152.

Grimm, K.J. (2007). Multivariate longitudinal methods for studying developmental relationships between depression and academic achievement. International Journal of Behavioral Development, 31(4), 328-339.

Grimm, K.J. and Ram, N. (2009). A second-order growth mixture model for developmental research. Research in Human Development, 6(2-3), 121-143. doi.org/10.1080/15427600902911221.

Hall, C.B., Lipton, R.B., Sliwinski, M. and Stewart, W.F. (2000). A change point model for estimating the onset of cognitive decline in preclinical Alzheimer's disease. Statistics in Medicine, 19(11-12), 1555-1566. doi:10.1002/(sici)1097-0258(20000615/30)19:11/12<1555::aid-sim445>3.0.co;2-3.

Hauser, R.M. and Weir, D. (2010). Recent developments in longitudinal studies of ageing in the United States. Demography, 47, S111-130. doi:10.1353/dem.2010.0012.

Hayden, K., Reed, B., Manly, J., Tommet, D., Pietrzak, R., Chelune, G., Yang, F., Revell, A., Bennett, D. and Jones, R. (2011). Cognitive decline in the elderly: an analysis of population heterogeneity. Age and Ageing, 40(6), 684-689. doi.org/10.1093/ageing/afr101.

Hofer, S.M., Gray, K.M., Piccinin, A.M., Mackinnon, A., Bontempo, D.E., Einfeld, S.L., Hoffman, L., Parmenter, T. and Tonge, B.J. (2009). Correlated and coupled within-person change in emotional and behavioral disturbance in individuals with intellectual disability. American Journal on Intellectual and Developmental Disabilities, 114(5), 307-321. doi:10.1352/1944-7558-114.5.307.

Hofer, S.M. and Piccinin, A.M. (2009). Integrative data analysis through coordination of measurement and analysis protocol across independent longitudinal studies. Psychological Methods, 14(2), 150-164. doi:10.1037/a0015566.

Hofer, S.M. and Piccinin, A.M. (2010). Toward an integrative science of life-span development and ageing. Journals of Gerontology: Series B, 65B(3), 269-278. doi:10.1093/geronb/gbq017.

Hoffman, L. (2015). Longitudinal analysis: modeling within-person fluctuation and change. New York: Routledge.

Hoffman, L. and Stawski, R. (2009). Persons as contexts: evaluating between-person and within-person effects in longitudinal analysis. Research in Human Development, 6(2-3), 97-120. doi:10.1080/ 15427600902911189.

Hoogendijk, E., Deeg, D., Poppelaars, J., van der Horst, M., Broese van Groenou, M., Comijs, H., Pasman, H., van Schoor, N., Suanet, B., Thomése, F., van Tilburg, T., Visser, M. and Huisman, M. (2016). The longitudinal ageing study Amsterdam: cohort update 2016 and major findings. European Journal of Epidemiology, 31(9), 927-945. doi:10.1007/s10654-016-0192-0.

Hox, J.J. (2010). Multilevel analysis: techniques and applications. 2nd ed. New York: Routledge.

Huisman, M., Poppelaars, J., van der Horst, M., Beekman, A.T., Brug, J., van Tilburg, T.G. and Deeg, D.J. (2011). Cohort profile: the longitudinal ageing study Amsterdam. International Journal of Epidemiology, 40(4), 868-876. doi:10.1093/ije/dyq219.

Hultsch, D.F., Hertzog, C., Dixon, R.A. and Small, B.J. (1998). Memory change in the aged. Cambridge: Cambridge University Press.

Johansson, B., Hofer, S.M., Allaire, J.C., Maldonado-Molina, M., Piccinin, A.M., Berg, S., Pedersen, H. and McClearn, G.E. (2004). Change in memory and cognitive functioning in the oldest-old: the effects of proximity to death in genetically related individuals over a six-year period. Psychology and Ageing, 19, 145-156. 
Johnson, D.K., Storandt, M., Morris, J.C. and Galvin, J.E. (2009). Longitudinal study of the transition from healthy ageing to Alzheimer disease. Archives of Neurology, 66(10), 1254-1259. doi:10.1001/archneurol.2009.158.

Jung, T. and Wickrama, K.A.S. (2008). An introduction to latent class growth analysis and growth mixture modeling. Social and Personality Psychology Compass, 2(1), 302-317. doi:10.1111/ j.1751-9004.2007.00054.x.

Karr, J.E., Graham, R.B., Hofer, S.M. and Muniz-Terrera, G. (2018). When does cognitive decline begin? A systematic review of change point studies on accelerated decline in cognitive and neurological outcomes preceding mild cognitive impairment, dementia, and death. Psychology and Ageing, 33(2), 195-218. doi:10.1037/pag0000236.

Kryscio, R.J., Abner, E.L., Lin, Y., Cooper, G.E., Fardo, D.W., Jicha, G.A., Nelson, P.T., Smith, C.D., van Eldik, L.J., Wan, L. and Schmitt, F.A. (2013). Adjusting for mortality when identifying risk factors for transitions to mild cognitive impairment and dementia. Journal of Alzheimer's disease: JAD, 35(4), 823-832. doi:10.3233/JAD-122146.

Laird, N.M. and Ware, J.H. (1982). Random-effects models for longitudinal data. Biometrics, 38(4), 963-974. doi:10.2307/2529876.

Lindwall, M., Cimino, C.R., Gibbons, L.E., Mitchell, M.B., Benitez, A., Brown, C.L., Kennison, R.F., Shirk, S.D., Atri, A., Robitaille, A., MacDonald, S.W.S., Aelinski, E.M., Willis, S.L., Schaie, K.W., Johansson, B., Praetorius, M., Dixon, R.A., Mungas, D.M., Hofer, S.M. and Piccinin, A.M. (2012). Dynamic associations of change in physical activity and change in cognitive function: coordinated analyses of four longitudinal studies. Journal of Ageing Research. doi:10.1155/2012/493598.

Liu, M. and Hancock, G. (2014). Unrestricted mixture models for class identification in growth mixture modeling. Educational and Psychological Measurement, 74(4), 557-584. doi:10.1177/ 0013164413519798.

McClearn, G.E., Johansson, B., Berg, S., Pedersen, N.L., Ahern, F., Petrill, S.A. and Plomin, R. (1997). Substantial genetic influence on cognitive abilities in twins 80 or more years old. Science, 276(5318), 1560-1563.

MacDonald, S.W.S., Hultsch, D.F., Strauss, E. and Dixon, R.A. (2003). Age-related slowing of digit symbol substitution revisited: what do longitudinal age changes reflect? Journals of Gerontology: Series B, 58(3), P187-P194.

Martin, M. and Hofer, S.M. (2004). Intraindividual variability, change, and ageing: conceptual and analytical issues. Gerontology, 50(1), 7-11. doi:10.1159/000074382.

Mitchell, M.B., Cimino, C.R., Benitez, A., Brown, C.L., Gibbons, L.E., Kennison, R.F., Shirk, S.D., Atri, A., Robitaille, A., MacDonald, S.W.S., Lindwall, M., Zelinski, E.M., Willis, S.L., Chaie, W., Johansson, B., Dixon, R.A., Mungas, D.M., Hofer, S. and Piccinin, A.M. (2012). Cognitively stimulating activities: effects on cognition across four studies with up to 21 years of longitudinal data. Journal of Ageing Research. doi:10.1155/2012/461592.

Molenaar, P.C. and Campbell, C.G. (2009). The new person-specific paradigm in psychology. Current Directions in Psychological Science, 18, 112-117. http://dx.doi.org/10.1111/j.1467-8721.2009.01619.x.

Muniz-Terrera, G.H. and Hall, C.B. (2015). Change point models. In S.K. Whitbourne (ed.), The encyclopedia of adulthood and ageing. Chichester, UK: John Wiley \& Sons.

Muniz-Terrera, G., Robitaille, A., Kelly, A., Johansson, B., Hofer, S. and Piccinin, A. (2016). Latent growth models matched to research questions to answer questions about dynamics of change in multiple processes. Journal of Clinical Epidemiology. doi:10.1016/j.jclinepi.2016.09.001.

Muniz-Terrera, G., van den Hout, A., Piccinin, A.M., Matthews, F.E. and Hofer, S.M. (2013). Investigating terminal decline: results from a UK population-based study of ageing. Psychology and Ageing, 28(2), 377-385. doi:10.1037/a0031000.

Muniz Terrera, G., van den Hout, A. and Matthews, F.E. (2011). Random change point models: investigating cognitive decline in the presence of missing data. Journal of Applied Statistics, 38(4), 705-716. doi:10.1080/02664760903563668.

Muthén, B.O. (2002). Beyond SEM: general latent variable modeling. Behaviormetrika, 29(1), 81-117. doi:10.2333/bhmk.29.81. 
Muthén, B. (2004). Latent variable analysis: growth mixture modeling and related techniques for longitudinal data. In: D. Kaplan (ed.), The SAGE handbook of quantitative methodology for the social sciences. Thousand Oaks, CA: SAGE Publications, Inc.

Muthén, B. and Shedden, K. (1999). Finite mixture modeling with mixture outcomes using the EM algorithm. Biometrics, 55(2), 463-469. doi:10.1111/j.0006-341X.1999.00463.x.

Nagin, D.S. (1999). Analyzing developmental trajectories: a semiparametric, group-based approach. Psychological Methods, 4(2), 139-157. doi:10.1037/1082-989X.4.2.139.

Nylund, K.L., Asparouhov, T. and Muthén, B.O. (2007). Deciding on the number of classes in latent class analysis and growth mixture modeling: a Monte Carlo simulation study. Structural Equation Modeling: A Multidisciplinary Journal, 14(4), 535-569. doi:10.1080/10705510701575396.

Pedersen, N.L., Lichtenstein, P. and Svedberg, P. (2002). The Swedish Twin Registry in the third millennium. Twin Research, 5(5), 427-432. doi:10.1375/136905202320906219.

Piccinin, A.M., Muniz-Terrera, G., Clouston, S., Reynolds, C.A., Thorvaldsson, V., Deary, I.J., Deeg, D.J.H., Johansson, B., Mackinnon, A., Spiro, A. III, Starr, J.M., Skoog, I. and Hofer, S.M. (2013). Coordinated analysis of age, sex, and education effects on change in MMSE scores. Journals of Gerontology: Series B, 68(3), 374-390. doi:10.1093/geronb/gbs077.

Praetorius Björk, M., Johansson, B. and Hassing, L.B. (2016). I forgot when I lost my grip-strong associations between cognition and grip strength in level of performance and change across time in relation to impending death. Neurobiology of Ageing, 38, 68-72. doi:10.1016/j.neurobiolageing.2015.11.010.

Proust, C. and Jacqmin-Gadda, H. (2005). Estimation of linear mixed models with a mixture of distribution for the random effects. Computer Methods and Programs in Biomedicine, 78(2), 165-173. doi:org/10.1016/j.cmpb.2004.12.004.

Robitaille, A., Garcia, L. and McIntosh, C. (2015). Joint trajectories of cognitive functioning and challenging behavior for persons living with dementia in long-term care. Psychology and Ageing, 30(3), 712-726. doi:10.1037/a0039333.

Robitaille, A., Muniz, G., Piccinin, A.M., Johansson, B. and Hofer, S.M. (2012). Multivariate longitudinal modeling of cognitive ageing: associations among change and variation in processing speed and visuospatial ability. GeroPsych (Bern), 25(1), 15-24. doi:10.1024/1662-9647/a000051.

Robitaille, A., Piccinin, A.M., Hofer, S.M., Johansson, B. and Muniz Terrera, G. (2018a). An examination of the heterogeneity in the pattern and association between rates of change in grip strength and global cognition in late life. A multivariate growth mixture modelling approach. Age and Ageing, 47(5), 692-697. doi:10.1093/ageing/afy048.

Robitaille, A., van den Hout, A., Machado, R. J. M., Bennett, D. A., Čukić, I., Deary, I.J., Hofer, S.M., Hoogendijk, E.O., Huisman, M., Johansson, B., Koval, A.V., van der Noordt, M., Piccinin, A.M., Rijnhart, J.J.M., Singh-Manoux, A., Skoog, J., Skoog, I., Starr, J., Vermunt, L., Clouston, S. and Muniz Terrera, G. (2018b). Transitions across cognitive states and death among older adults in relation to education: a multistate survival model using data from six longitudinal studies. Alzheimer's and Dementia, 14(4), 462-472. doi:10.1016/j.jalz.2017.10.003.

Singer, J.D. and Willett, J.B. (2003). Applied longitudinal data analysis: modeling change and event occurrence. New York: Oxford University Press.

Sliwinski, M. and Buschke, H. (1999). Cross-sectional and longitudinal relationships among age, memory and processing speed. Psychology and Ageing, 14, 18-33.

Sliwinski, M. and Buschke, H. (2004). Modeling intraindividual cognitive change in ageing adults: results from the Einstein Ageing Studies. Ageing, Neuropsychology and Cognition, 11, 196-211.

Sliwinski, M. and Mogle, J. (2008). The multiple facets of change: implications for modeling relationships in cognitive ageing research. In: S.M. Hofer and D.F. Alwin (eds), Handbook on cognitive ageing: interdisciplinary perspectives. Thousand Oaks, CA: Sage Publications.

Sliwinski, M.J., Hofer, S.M. and Hall, C. (2003). Correlated and coupled cognitive change in older adults with and without preclinical dementia. Psychology and Ageing, 18(4), 672-683. doi:10.1037/0882-7974.18.4.672.

Small, B. and Bäckman, L. (2007). Longitudinal trajectories of cognitive change in preclinical Alzheimer's disease: a growth mixture modeling analysis. Cortex, 43(7), 826-834. doi:org/10.1016/ S0010-9452(08)70682-8. 
Sonnega, A., Faul, J.D., Ofstedal, M.B., Langa, K.M., Phillips, J.W. and Weir, D.R. (2014). Cohort profile: the Health and Retirement Study (HRS). International Journal of Epidemiology, 43(2), 576-585. doi:10.1093/ije/dyu067.

Terrera, G., Brayne, C. and Matthews, F. (2010). One size fits all? Why we need more sophisticated analytical methods in the explanation of trajectories of cognition in older age and their potential risk factors. International Psychogeriatrics, 22(2), 291-299. doi.org/10.1017/S1041610209990937.

Terrera, G.M., Minett, T., Brayne, C. and Matthews, F. E. (2014). Education associated with a delayed onset of terminal decline. Age and Ageing, 43(1), 26-31. doi:10.1093/ageing/aft150.

Thorvaldsson, V., Hofer, S.M., Berg, S., Skoog, I., Sacuiu, S. and Johansson, B. (2008). Onset of terminal decline in cognitive abilities in individuals without dementia. Neurology, 71(12), 882-887. doi:10.1212/01.wnl.0000312379.02302.ba.

van den Hout, A. (2017). Multi-state survival models for interval-censored data. Boca Raton, FL: Taylor \& Francis.

Wang, L. and Maxwell, S.E. (2015). On disaggregating between-person and within-person effects with longitudinal data using multilevel models. Psychological Methods, 20(1), 63-83. doi:10.1037/ met0000030.

Woodard, J.L. (2017). A quarter century of advances in the statistical analysis of longitudinal neuropsychological data. Neuropsychology, 31(8), 1020. doi:10.1037/neu0000386.

Xie, H., Mayo, N. and Koski, L. (2011). Identifying and characterizing trajectories of cognitive change in older persons with mild cognitive impairment. Dementia and Geriatric Cognitive Disorders, 31(2), 165-172. doi.org/10.1159/000323568.

Zammit, A.R., Piccinin, A.M., Duggan, E.C., Koval, A., Clouston, S., Robitaille, A., Brown, C.L., Handschuh, P., Wu, C., Jarry, V., Finkel, D., Graham, R.B., Muniz-Terrera, G., Björk, M.P., Bennett, D., Deeg, D.J., Johansson, B., Katz, M.J., Kaye, J., Lipton, R.B., Martin, M., Pederson, N.L., Spiro, A., Zimprich, D. and Hofer, S.M. (2019). A coordinated multi-study analysis of the longitudinal association between handgrip strength and cognitive function in older adults. Journals of Gerontology: Series B. doi:10.1093/geronb/gbz072. 